\title{
La religión como currículo social: educación, valores e Islam en Europa
}

Vicente Javier Llorent García'

Carolina Ivanescu"

\section{Resumen}

Cuando la religión se convierte en parte integrante de los debates políticos modernos, las ideas religiosas influyen en las decisiones y definiciones de conceptos como la educación, la participación en el mercado laboral, la estructura y función de la familia como unidad social y la igualdad. La integración y la inmigración pasan a primer plano a medida que el concepto de ciudadanía adquiere dimensiones culturalistas y moralizantes. Finalmente, religión, política y educación se interfieren en las áreas de la vida que pertenecen a la esfera privada y pública. Desde nuestra perspectiva pedagógica, entendemos que las líneas educativas marcadas por la religión influyen notablemente en el devenir de muchos ciudadanos y se incrustan en el foro político-social. Así nos encontramos que la religión como poderoso instrumento de socialización ejerce una gran influencia intencionada en la educación y valores de los ciudadanos y se configura como el currículo social, normalmente oculto, del contexto próximo de muchos ciudadanos. La presencia del Islam y los musulmanes en Europa, aunque no es un fenómeno nuevo, es cuestionada por los Estados-nación confrontando el currículo oficial, la educación reglada, con el currículo social, muy influido por el Islam entre sus fieles. De esta manera se convierte en parte integrante de la lucha por la identidad nacional individual y colectiva, ya sea en lo referente al laicismo, la democracia o ciudadanía. Y en este sentido, queremos aportar una revisión actualizada de esta cuestión tan presente en Europa.

\section{Palabras Clave}

Religión - Educación - Política - Musulmanes - Inmigración.

I- Universidad de Córdoba, Córdoba, 


\title{
Religion as social curriculum: education, values and Islam in Europe
}

Vicente Javier Llorent García'

Carolina Ivanescu"

\begin{abstract}
When religion is converted in an integral part of modern political debates, religious ideas influence the decisions and definitions of concepts such as education, the participation in the labor market, the structure and function of the family as a social unit, and the equality. Integration and immigration come to the forefront as the concept of citizenship acquires culturalist and moralizing dimensions. Finally, religion, politics and education begin to interfere in the areas of life that belong to the private and public spheres. From our pedagogical perspective we understand that the educational lines marked by religion influence remarkably the future of many citizens, and attach themselves to the social-political realm. We thus become aware of the fact that religion as a powerful instrument of socialization exerts a strong influence upon citizens' education and values, and presents itself as the - normally hidden - social curriculum of the context closer to many citizens. The presence of Islam and of Muslims in Europe, though not a new phenomenon, is questioned by the nation-states, confronting the official curriculum and the regulated education, with the social curriculum, strongly influenced by Islam among its followers. It therefore converts itself in an integral part of the struggle for individual and collective national identity, be it with respect to laity, to democracy or to citizenship. And, in this sense, we would like to present an up-to-date review of this question so present in Europe today.
\end{abstract}

\section{Keywords}

Religion - Education - Politics - Muslims - Immigration.

I- Universidad de Córdoba, Córdoba, Spain Contact: villorent@uco.es.

II- Universidad Erasmus, Rotterdam, The Netherlands.

Contact: ivanescu@fsw.eur.nl 
Educación y cultura -en cuanto estructura determinada de las instituciones, medios y fines- contribuyen poderosamente a garantizar la continuidad y la perpetuación de la etnicidad de un grupo social concreto. La educación puede, en efecto, proporcionar una importante línea de continuidad con el pasado que, con frecuencia, se mantiene viva a través de la práctica de las tradiciones culturales. Dentro de una misma sociedad existen, sin embargo, grandes desigualdades, tanto en acceso a la educación como en las prácticas culturales, que provocan un impacto diferente en los niños y los jóvenes. Si bien podemos identificar un currículo oficial donde, a través de leyes y otros textos legislativos, se plasman las intenciones educativas del Estado y en definitiva el modelo antropológico de cada sociedad; también debemos de ser conscientes de que la educación y cultura del contexto social del individuo suponen un currículo más desapercibido y sutil. Así nos encontramos que la religión como poderoso instrumento de socialización ejerce una gran influencia intencionada en la educación y valores de los ciudadanos y se configura como el currículo social, normalmente oculto, del contexto próximo de muchos ciudadanos (LLORENT; LLORENT, 2009).

La presencia del Islam y los musulmanes en Europa, aunque en esencia no es un fenómeno nuevo, es cuestionada por los Estados-nación de tal manera, al confrontar el currículo oficial, la educación reglada, con el currículo social, muy influido por el Islam entre sus fieles. De esta manera se convierte en parte integrante de la lucha por la identidad nacional individual y colectiva, ya sea en lo referente al laicismo, la democracia o ciudadanía. La preocupación por el creciente número de musulmanes y la creciente importancia del Islam en los Estadosnación de Europa occidental se ven favorecidas por su presencia desde hace décadas en la gestión de la minoría estados-nación contemporáneos. Estos problemas sufrieron un giro especial por acontecimientos como el 11-S (atentado terrorista en EEUU en 2001),
11-M (atentado terrorista en España en 2004), 7-J (atentado terrorista en Inglaterra en 2005) y el asesinato de Theo van Gogh. (TILLY, 2006; RAI, 2006; BURUMA, 2006; EYERMAN, 2008). Las dinámicas en torno a la nueva religión de los inmigrantes, que se considera el Islam, son una oportunidad para echar un vistazo a la complicada relación de los estados laicos de Europa occidental con la religión (IVANESCU, 2010).

\section{Los estados-nación, entre las normas y los valores}

El Estado-nación moderno se concibe como una unidad política laica o en otros casos no confesional, donde el poder se distribuye entre las personas en un estado independiente controlado por el mecanismo de la ciudadanía (GELLNER, 1983; ANDERSON, 1991; HOBSBAWM, 1990). Esta unidad se concibe como culturalmente homogénea (ANDERSON, 1991; CALHOUN, 1994). Desde la perspectiva del Estado como agente, el origen étnico, la especificidad cultural y la religión se "desestiman como una característica de sociedades 'simples' y 'pre-políticas', o de grupos marginales que deben integrarse en el Estado-nación" (HUTCHINSON, 2000, p. 653).

Como legado de la filosofía de la Ilustración y los acuerdos específicos a nivel nacional de laicidad, la religión se considera opuesta a la razón y a la racionalidad, se considera como algo separado de la esfera política y se sitúa en la vida privada de las personas. El principal paradigma a través del cual la ciencia social ha estudiado la religión es la teoría de la secularización. Casanova (1994) distinguió tres direcciones diferentes que la teoría de la secularización ha tenido: la diferenciación de la esfera laica de la religión, la secularización como un declive de la religión (prácticas y creencias) y la secularización como la privatización de la religión a su propia esfera. Él acepta la diferenciación dentro de la sociedad occidental, pero muestra que las instituciones religiosas siguen desempeñando 
un papel importante, sobre todo dentro de la sociedad civil. Esto no se opone necesariamente al proceso de secularización. Sengers (2005) afirma que la secularización y la transformación de la religión pueden ser procesos simultáneos. La secularización en todos los aspectos de la sociedad y la transformación de la religión evidente en el número de asociaciones religiosas y su forma de tratar con la sociedad, y el número y la forma de trabajar de las organizaciones no occidentales pueden ir de la mano (DOBBELAERE, 2002).

La construcción de Estados-nación europeos supuso en muchos casos, la atribución, incluso forzada, de una lengua nacional, una cultura nacional y unas instituciones nacionales. En este contexto, los migrantes por definición, eran vistos como los otros, que por el hecho de cruzar las fronteras de la naciónestado reforzaron el significado de dichas fronteras. Los demás de la nación-estado son vistos como invitados con un objetivo (trabajo, ocio etc.) y se espera que regresen, a no ser que se nacionalicen. Pero incluso cuando se nacionalizan, se hacen parte de una nación, el tema de la diferencia sigue siendo doloroso. La misma diferencia que se encuentra en la base de la identidad es también la base de la diferencia (CONNOLLY, 1991). No hay que olvidar que los ciudadanos suelen ser habitantes, los miembros de asociaciones de poder universales y particulares, entre los que el Estado-nación no es más que una (HAMMAR, 1986). Otras asociaciones podrían ser muy bien definidas en términos de religión, que puede ser nacional o de carácter transnacional (ROY, 2004). El dilema de la diferencia, el dilema de negar o afirmar la diferencia en lo referente a la igualdad y la pluralidad en el concepto de ciudadanía, está lejos de ser resuelto (YOUNG, 1990).

La migración y la formación de las minorías representan retos para el Estado-nación. En el caso de los inmigrantes/minorias de origen musulmán, la tensión se puede identificar a varios niveles, de los cuales nos gustaría destacar dos: las desigualdades socio-económicas que son propios del proceso de la migración laboral y el desafío que el resurgimiento de la religión representa para el Estado laico. Estas tensiones se producen en el contexto de cambios más generales en la política de integración en Europa, es decir, la localización de la formulación de políticas, la transformación de las ciudades complacientes en ciudades exigentes, la combinación de la integración con la inmigración $\mathrm{y}$, finalmente, el papel importante de la agenda de integración en el partidismo (GUIRAUDON; LAHAV, 2006).

Desde el punto de vista del Estado laico, la migración se presenta como un desafío por la posibilidad de un resurgimiento religioso que pueda presentar. La presencia religiosa puede ser vista como una doble amenaza: en primer lugar, como pluralismo cultural y en segundo lugar, como una amenaza para el Estado laico. Sin embargo, la cuestión es más complicada. En la raíz de esta doble tensión se encuentra la construcción del Estado moderno en todo el proceso de secularización. Hay una fuerte conexión entre la teoría de la secularización, el discurso ideológico de la formación del Estado y los discursos racionalistas sobre la modernidad (HADDEN, 1987; STARK; FINKE, 2000). El laicismo necesita un sustituto para la base ideológica de la religión y lo encuentra en el nacionalismo (ANDERSON, 1991).

La interacción entre política y religión no deja intacto a ninguno. De hecho, los dos nunca han estado completamente separados. Aunque en la actualidad pensamos en el laicismo como la separación de poderes entre el Estado y las instituciones religiosas, en detrimento de estas últimas (WILSON; SOUTH PLACE ETHICAL SOCIETY, 1996), según señala Asad, lo laico y lo religioso son interdependientes. Asad atrae nuestra atención hacia los hechos históricos que hay detrás de la conexión entre los dos conceptos, a saber, que mientras que lo laico fue inicialmente una parte del discurso teológico (saeculum), lo religioso se construye con los discursos políticos y científicos como la diferenciación 
a través del desempeño del organismo de lo laico y de lo religioso (ASAD, 2003).

El Islam permite una identificación personal y colectiva alternativa con respecto al Estado, y parafraseando a Mahmood (2005), a través de la posibilidad de una política de piedad que presenta un posible desafío a las formas de acción propuestas por el principio de ciudadanía. Al mismo tiempo, los ideales y valores islámicos propuestos como directrices para la buena ciudadanía, y por tanto pertenencia a la nación-estado, ofrecen una alternativa, si no una visión radicalmente diferente de los valores considerados universales para el Estado-nación.

En este artículo, el término religionalización se ha utilizado para describir dos tipos de fenómenos relacionados con la religión. En primer lugar, tiene que ver con la falta de una frontera clara entre los ámbitos político y religioso (ver también MARTIN, 1978, en el objetivo común de los dos), y en segundo lugar, se utiliza como una deconstrucción de la objetividad y la universalidad de los valores políticamente nacionalizados. Al utilizar el término de religionalización, nos gustaría señalar que las creencias y los valores percibidos como algo natural y universal son tan socialmente construidos y son una cuestión tan de convención y creencia social como la propia religión. Por otra parte, los discursos de la unificación de la nación usan el mecanismo de la sacralización con el fin de ganar autoridad. A medida que los sentimientos nacionalistas aumentan en la población y en el discurso político, los valores que promueve el Estado-nación adquieren, a través de actos rituales de pertenencia, un aura de santidad, esencial para la identidad individual y colectiva de los ciudadanos.

\section{La religión en el currículo social:} el Islam en Holanda

En esta sección, nos referiremos a la forma en que la religión, ayudada por la preocupación sobre la inmigración, se politiza articulando un currículo social. La tragedia multicultural, un término acuñado por el publicista Scheffer (2000) en su influyente artículo que apareció en los medios de comunicación nacionales, es probablemente la mejor descripción de cómo se percibe y se maneja el tema del Islam y los musulmanes. El supuesto básico era el de la incompatibilidad entre los valores occidentales y los islámicos.

Sin embargo, esto no era nuevo en la política nacional holandesa. La misma situación fue adoptada libremente por los políticos influyentes, como Bolkestein, el líder del Partido Popular para la Libertad y la Democracia (Volkspartij voor Vrijheid en Democratie, VVD) de corte liberal, ya en el comienzo de la década de 1990. A finales de la década de 1990 , se predijo la relación existente entre la incompatibilidad de culturas y un drama socio-cultural y tal vez hasta cierto punto, se creó como una posibilidad en la conferencia de Paul Schnabel, el director de la Oficina Social y Cultural de Planificación (Sociaal en Cultureel Planbureau, SCP), agencia nacional de investigación, que fue de la opinión de que la integración de los inmigrantes podría no funcionar.

Lo más importante tal vez es la visión esencialista de la cultura, que es vista como un bloque monolítico de normas y valores que determinan la (presumiblemente homogénea) forma de vida de ciertos grupos y/o individuos. Esta manera de ver la cultura, de inmediato se polariza, ya que se supone que hay una cultura holandesa inmutable y una cultura musulmana que se oponen, no se comunican, y no se influyen entre sí (DUYVENDAK; PELS; RIJKSCHROEFF, 2009).

El Islam como tema de controversia abierta y debate político salió a la luz con PimFortuyn, el líder carismático de la Rotterdam Habitable (Leefbaar Rotterdam, LR), el partido populista de derechas. Su discurso se basaba en la polarización de los holandeses nativos y los inmigrantes, y una promoción activa del Islam como el principal problema, ya que el Islam es una cultura atrasada. La hipótesis principal fue que los inmigrantes musulmanes (no integrados) son el principal problema de los Países Bajos, su presencia y su actitud ponen 
en peligro los logros y placeres de Occidente. El logro de LR fue convertirse en el partido más importante de la ciudad de Rotterdam en el período comprendido entre 2002 y 2006. Después del asesinato de Fortuyn en 2002, LR continuó empujando una agenda política con el tema central del Islam.

El problema con el Islam se define como la tensión existente entre los valores y normas islámicos y occidentales, y la tensión entre las culturas arraigadas en las tradiciones y las culturas arraigadas en la modernidad. Sin embargo, la distinción entre la tradición y la modernidad no es inocente, sino que está profundamente inmersa en la lucha por el poder y la supremacía. El debate representa que los países occidentales son, sin duda, modernos, y los Países Bajos se presentan como individualistas y laicos (GEMEENTE ROTTERDAM, 2004). Los musulmanes representan el mantenimiento de sus raíces en la religión y la tradición, y por lo tanto se muestra que no son capaces de pasar el umbral de la modernidad, lo que requiere distancia de la religión como requieren los principios del laicismo, y la relativización de las normas y valores tradicionales como promueven las ideas democrático-liberales.

El debate político se inició con los expertos invitados y representantes de la población musulmana, que, de acuerdo con las prácticas democráticas, estuvieron involucrados en la discusión. La forma en que se celebraron las reuniones de expertos sólo consiguió reforzar la percepción de que el Islam es la causa de los problemas de integración. La religión, junto con la cultura, recibió toda la culpa por el mal estado de la integración. Los debates internos terminaron estando repletos de consejos y de la definición del problema. Por otra parte, al señalar la importancia de los debates públicos por encima de los internos, fue el escenario de acción que se consideraba más importante la interacción pública entre musulmanes y no musulmanes, o rotterdameses autóctonos.

El objetivo de los debates internos era eliminar tanto como fuera posible "ideas erróneas y la ignorancia" en la comunidad musulmana (GEMEENTE ROTTERDAM, 2004, p. 9). Los debates internos fueron una serie de seminarios y encuentros destinados sólo para la comunidad musulmana, que tenía por objeto aclarar la posición, objetivos y ambiciones de los musulmanes en la sociedad y sus puntos de vista acerca de la contribución civil y la participación. Al mismo tiempo, los debates fueron dirigidos directamente hacia los temas delicados, mostrándose también el punto de vista del municipio sobre lo que era el problema y cómo tenía que resolverse. Entre los temas delicados estaba la posición de las instituciones religiosas en la sociedad, es decir, que las mezquitas, el trabajo de los imanes y las auto-organizaciones actúan como obstáculos a la integración, lo que provoca separatismo social. En otras palabras, las organizaciones religiosas se perciben como lugares posibles para el comunitarismo religioso o étnico. Como solución, las instituciones religiosas, se argumentó, deberían estar abiertas a la sociedad en general y también deberían convertirse en centros sociales, además de religiosos. Parte de la solución era oponerse a la desigualdad de género dentro de las instituciones religiosas y cambiar el hecho de que las mezquitas eran exclusivas del mundo de los hombres, en términos de visitas y de poder de decisión (GEMEENTE ROTTERDAM, 2005). En este caso, vemos un intento de intervenir en las funciones sociales de las instituciones religiosas y en la jerarquía social de las creencias y organizaciones religiosas.

La política intervencionista del Estado a nivellocal tambiénse puedeseguiren relación con el tema de la emancipación. La declaración "los musulmanes han de emanciparse renunciando a su religión" (GEMEENTE ROTTERDAM, 2004, p. 13) provocó una acalorada discusión. El discurso de la emancipación relacionado con el tema de la religión no es algo nuevo en los Países Bajos. Como señalaremos en los siguientes capitulos, uno de los factores que contribuyen a la disminución de pilarización ${ }^{1}$

1- Pillarizationen inglés - verzuilingen holandés, término acuñado en Holanda y Bélgica, que hace referencia a la concentración de poder, en 
es la fructuosa implementación del proceso de emancipación que hace que el aislamiento y la diferenciación sean redundantes. En el proceso de emancipación, las diferencias se nivelan hacia abajo, diferencias que son importantes desde el punto de vista de la igualdad social. Desde el punto de vista del Estado laico, la diferencia importante que debe ser destruida es la de las creencias, normas y valores que difieren de los promovidos por el Estado.

El renovado interés y preocupación por la religión no aparece en un vacío. Con el fin de comprender la dinámica que facilitó el éxito de Pim Fortuyn, LR y su programa centrado en el Islam, es fundamental que tengamos una comprensión más profunda de las formas en que el Estado holandés ha abordado la religión y la inmigración. Para ello, es importante seguir la intersección de la tradición de pilarización específica de la zona, con su compromiso especial de laicismo a través de la institucionalización pública de la religión, y la forma en que el trato de igualdad y diferencia a través del principio de ciudadanía se convirtió en tiempo.

\section{Inmigración, educación, multiculturalidad y diversidad religiosa}

A pesar de que el historial de inmigración de los Países Bajos comenzó en la época postcolonial, el discurso sobre ella está dominado por una narrativa iniciada a partir de la ola de inmigración laboral, la llegada de inmigrantes sin cualificación profesional a finales de la década de los 50, inicialmente desde España e Italia, después de países musulmanes como Turquía y Marruecos (WILTERDINK, 1998). En este punto, la inmigración es una solución económica a corto plazo de la lucha con las consecuencias de la Segunda Guerra Mundial. Con el fin de darles cabida, se toman medidas para asegurar que los grupos de inmigrantes

un Pilar, constructo social que aglutina a grupos que comparten valores 0 creencias, como los protestantes, católicos, socialdemócratas; que tienen su propios partidos, asociaciones, locales, periódicos, escuelas, hospitales. mantengan su propia cultura como un recuerdo que evitará problemas cuando regresen a sus respectivos países de origen (ENTZINGER, 1984). Los inmigrantes son vistos como invitados temporales, y como tales, su integración no es un problema. La cultura no se considera un problema, los grupos en cuestión son vistos como transitorios para el Estado. En este periodo, el alojamiento de los grupos (étnicos) significa el reconocimiento de la auto-identificación y de procesos de auto-organización.

Esta forma de auto-organización se apoya en la estructura de alojamiento existente, conocida como pilarización (LIJPHART, 1968). En los Países Bajos, el multiculturalismo se basó en los mecanismos institucionales existentes para subvencionar a las organizaciones políticas o religiosas. La tolerancia de las prácticas religiosas tenían poco que ver con las estrategias nacionales de integración (KOOPMANS, 2005).

En calidad de invitada, la mano de obra migrante tenía derecho e incluso se le animó a preservar su identidad social, cultural y religiosa, con el fin de facilitar el traslado cuando fuera necesario. Este enfoque pragmático permitió una especie de pluralismo cultural a ser desarrollado, donde los diferentes grupos étnicos intentaron un sistema de auto-institucionalización a través de los grupos, asociaciones y organizaciones culturales y religiosas. Estos esfuerzos fueron alentados y sostenidos por las llamadas políticas multiculturales. Aunque los estudiosos piensan que el pluralismo es el efecto del intento de multiculturalismo de proteger la identidad de los trabajadores inmigrantes (SNIDERMANY; HAGENDOORN, 2007; KOOPMANS, 2005), el resultado no fue un baluarte musulmán o de los inmigrantes. Al separarse de la población holandesa, los inmigrantes se organizaron en grupos étnicos, con organizaciones culturales, sociales y religiosas específicas, apareciendo una gran cantidad de formaciones sociales fragmentadas (JOPPKE, 2004).

La integración individual, el nuevo enfoque, permite que el peso dela responsabilidad que hasta ahora presionaba los hombros del 
Estado sea transferido a la propia persona. Desde el punto de vista del Estado, el individuo puede ser clasificado con fines de gestión como un sistema o una unidad no integrada. Uno de los principales factores desencadenantes del cambio de un enfoque centrado en los grupos a otro centrado en los individuos fue la crítica de que el primer enfoque alentaba la separación de los inmigrantes (ENTZINGER, 1984), lo que en casos extremos, lleva al aislamiento y a la falta de cohesión social de la sociedad. La individualización del discurso fue de la mano con la culturalización. El discurso se relacionó con el bajo nivel educativo, el desempleo y la delincuencia en grupos étnicos específicos, dando lugar a una fuerte polarización y a la moralización de los socios de la discusión, reforzado por el desequilibrio de poder entre los dos. Entonces ahora sí, los inmigrantes, los ciudadanos de clase marginada de la sociedad holandesa según Ghorashi (2003), tienen la culpa de los problemas, con los que la sociedad estaba tratando en ese momento.

Los cambios en el discurso sobre la inmigración y la unión de la inmigración con la integración tuvieron un efecto en el concepto de ciudadanía. La preocupación por los aspectos culturales llevan al discurso culturalista (VAN DEN BERG; SCHINKEL, 2009) o la culturalización de la ciudadanía (DUYVENDAK; TONKENS; HURENKAMP, 2008). Este cambio tiene la consecuencia de que las diferencias económicas se consideran culturales. La integración es vista como un asunto individual, mientras que la cultura es definida como problemática y considerada la causa de otros problemas (SCHINKEL, 2008). A medida que la preocupación por la cultura poco a poco se centra en el conocimiento de las normas y los valores de la cultura holandesa y de lengua holandesa como un requisito previo del proceso para la integración, la ciudadanía adquiere una dimensión moral (HOUDT; SCHINKELY, 2009).

La categoría de los musulmanes que se utiliza en el discurso político y la política está estrechamente relacionada con el proclamado laicismo del Estado. Cuando se habla de adaptaciones nacionales del concepto de laicismo, Martin (1978) define los Países Bajos como el modelo 60:40, en alusión al modelo de organización social en general y el laicismo en particular. ${ }^{2}$ Él ve los pilares como las estructuras sociales con una doble función, por un lado, definir y mantener a la gente dentro de las organizaciones religiosas (iglesias), y por otro lado definir el mundo como laico fuera de los pilares. De esta manera, "hay una polarización a nivel de la cultura y de su sistema de significados que complementa la falta de polarización en el plano político" (MARTIN, 1978, p. 199). Este equilibrio sólo puede inclinarse por un renacimiento religioso. En su documento sobre la dimensión histórica y geográfica de la secularización en los Países Bajos, Knippenberg (1998) es de la opinión de que el aumento de la población musulmana e hindú como consecuencia de la migración y de sus altas tasas de fecundidad será un desafío para el proceso de secularización.

Sin embargo, como en este caso se señala, el desafío al laicismo provocado por la población musulmana surge a través del hueco excavado en la culturización y la moralización del principio de la ciudadanía y su interés posterior en la vida privada de las personas. Las reacciones de los musulmanes en el debate claramente refutan la universalidad de los valores democráticos occidentales liberales.

\section{Política, educación y religión islámica}

El debate del Islam y la integración introdujeron la religión en el debate público y político en Holanda. A través de esta iniciativa, temporal y parcial, la religión consigue una plataforma, una voz en la conversación

2- La pluralidad de las confesiones hace posible la adaptación a las diferentes actitudes políticas y niveles de estado. En el patrón intermedio 0 "mixto" aparecen dos cuasi-monopolios (protestantes contra católicos en la proporción 60:40) que están tratando de dominar la escena. Esta estructura obliga a cada uno a hacer compromisos con el fin de mantener la unidad, por lo tanto, emerge un modelo de tolerancia. 
democrática y una oportunidad de participar en el proceso político. El programa no sólo es puesto en marcha para definir y resolver la cultura y un problema, sino que también se construye en la oposición entre ellos (los musulmanes) y nosotros (los holandeses autóctonos). Aún así, esta oportunidad permite a los musulmanes que expongan un razonamiento en el ámbito político en nombre de la religión, lo que disminuye la distancia entre la política y la religión tal como propone el laicismo.

Los musulmanes deploran la excesiva atención hacia las diferencias culturales y religiosas. Al contrario que Martin (1978), los inmigrantes de la diáspora, en lugar de equiparar la religión con la cultura, tratan de diferenciarlas, dejando claro que se habla desde un punto de vista religioso. Los musulmanes se definen en contra de la tradición, la cultura y en contra de la memoria - produciendo así la ruptura entre la primera y segunda generación de inmigrantes (HERVIEU-LE'GER, 2002). Algunos argumentan que si hay un problema con la minoría musulmana, su origen no debe buscarse en el ámbito cultural o religioso, sino más bien en el ámbito socio-económico que no se está abordando. Las realidades de la inmigración: la menor posición socioeconómica y la discriminación son justificadas por su situación actual, pero no por la religión. Mientras que la categoría de la religión se utilizó en el discurso público a través de la frecuente referencia al Islam, hay una clara fusión de la cultura, el origen étnico y la religión.

Como ya hemos señalado con los ejemplos anteriores, durante los debates los representantes de la comunidad musulmana aceptaron fácilmente el colectivo que se les había asignado. Además, defendieron la religión de la confusión con la tradición y la cultura, y señalaron los valores comunes de la religión y la ciudadanía. Sin embargo, además de la discusión acerca de los valores, la verdad absoluta también se mencionó una vez: "somos responsables frente a Allah y no frente a los demás" (GEEMENTE ROTTERDAM, 2004, p. 15). Esta declaración proporciona un espacio para la reflexión sobre el fin último de la orientación de una sociedad armoniosa, para adherirse a ciertas normas y valores. $\mathrm{Si}$, para la persona religiosa, la lucha con los problemas del aquí y ahora tiene sentido en la perspectiva del diálogo con el Creador, ¿cómo pueden tenerse en cuenta los valores del Estado democrático liberal y la forma de tratar con el aquí y ahora?

La politización de la religión -aunque las razones permitidas y de carácter temporal que se dieron en el nombre de la religión, por parte de las personas que estaban representando o que se percibía que representaban a un grupo religioso- empezó a escucharse en los ámbitos público y político a través de un amplio rango de temas, de los cuales sólo unos pocos estaban bajo la jurisdicción laica de la religión. Através del proceso, facilitado por las prácticas democráticas apoyadas por el Estado-nación a nivel local y por los cambios en la comprensión del concepto de ciudadanía, la religión se politizó. La politización de la religión permitió la interpenetración entre las esferas de la religión y la política que se entienden como necesariamente separadas por el laicismo.

\section{Reflexiones finales}

La religionalización de la política, el efecto de la religión en la política, se puede apreciar en diferentes niveles. Una vez que la religión se desplaza al centro del debate político, las instituciones y actores que hablan en nombre del Islam lo hacen convirtiéndose así en participantes del debate. Como la religión se combina con la cultura y el origen étnico, los argumentos religiosos entran en discusiones que no necesariamente están en el ámbito de la religión, pero que son de índole social, económica o política. $\mathrm{Al}$ participar en las discusiones sobre temas que no necesariamente están bajo su jurisdicción, los argumentos se dan o se entienden como realizados en el nombre de 
la religión sobre una amplia gama de temas, tales como los objetivos y los medios de la educación (religiosa), la participación en el mercado laboral, la estructura familiar y roles de género, sólo por nombrar algunos (IVANESCU, 2010).

Por otra parte, es probable que los valores y normas que se presentan en estos argumentos difieran de las normas y valores que son promovidos por las democracias liberales occidentales, y que están en la base de la formación del Estado-nación europeo. Estos sistemas alternativos de referencia suponen un reto por la relativización de las normas y valores liberales que se dan por sentados y ofrecen una visión crítica hacia su aplicación en la práctica. Los argumentos e ideas religiosas están presentes en la esfera política y tienen la oportunidad de producir un impacto en las decisiones que son de naturaleza política.

Finalmente, la culturalización y la moralización de la ciudadanía y la interferencia de la política en las áreas de la vida que pertenecen a la esfera privada, o currículo social, como la familia, los niños y la educación, las creencias y la lealtad desdibujan aún más la separación entre lo privado y lo público, y entre política y religión. Esta influencia ad hoc politiza cada religión mientras que religionaliza la política, por lo que la diferenciación de las esferas no es tan clara como la teoría del laicismo podría desear.

La religión está volviendo a la esfera pública a través de las oportunidades brindadas por los cambios en la regulación de la inmigración y los procesos de integración, y por la remodelación de los modelos de ciudadanía. Como consecuencia de la interacción pública entre religión y política, ambos participantes se ven influidos por los demás. Mientras que la religión está politizada, la política también se religionaliza. La religión está protegiendo sus propios derechos y así, reestructura la discusión sobre la hegemonía del Estado oponiendo la universalidad de los valores democráticos del Occidente liberal.
La religión, según la teoría de la secularización está cada vez más marginada a la esfera privada y pierde su relevancia social. Sin embargo, a través de su presencia en la arena política, recibe atención pública y política y se posiciona en el centro de la esfera pública de nuevo. Esta atención se debe al cambio de actitud del Estado y la ciudad de Rotterdam con la población de origen inmigrante $\mathrm{y} / \mathrm{o}$ una experiencia religiosa diferente. Esto puede verse fácilmente como una inversión del pilar de la estabilidad, el centro de gravedad de un determinado momento histórico (TAMBIAH, 1990). Como la mayoría de las reglas y acuerdos de la religión son una compleja mezcla con el principio de ciudadanía y los problemas de acuerdos de las minorías, el proceso de revitalización de la religión puede entenderse como una estrategia para construir un otro nacional, cultural y religioso. A través de la polarización, el otro ayuda a construir una identidad nacional y el correspondiente concepto de ciudadanía.

La integración y la inmigración pasan al primer plano a medida que el concepto de ciudadanía adquiere una dimensión culturalista y moralizante, donde las normas y los valores se comparan entre sí. El Estado laico se preocupa sobre todo por aquellos aspectos de la cultura que parecen contradecir de alguna manera sus paradigmas y el equilibrio de poder. El Islam es visto por el Estado-nación como una posible amenaza, especialmente ya que por ser visible y público contradice uno de sus principios básicos, a saber: el secularismo. Sin embargo, los principios democráticos, que también están en el corazón de la nación-estado liberal y la importancia de la integración de los inmigrantes/minorías en la agenda política, ofrecen la posibilidad de que la religión se haga visible en el ámbito público y político. En este proceso, la religión se politiza, adquiere un aura pública y se convierte en sujeto y objeto de los procesos políticos y los discursos, contrayendo el currículo social que trasmite a las generaciones la educación y cultura ligadas al contexto actual. 


\section{Referencias}

ANDERSON, Benedict. Imagined communities: reflections on the origin and spread of nationalism. London: Verso, 1991.

ASAD, Talal. Formations of the secular: Christianity, Islam, modernity. Stanford: Stanford University Press, 2003.

BURUMA, lan. Murder in Amsterdam: the death of Theo van Gogh and the limits of tolerance. New York: Penguin, 2006.

CALHOUN, Craig J. Social theory and the politics of identity. Oxford: Blackwell, 1994.

CASANOVA, José. Public religions in the modern world. Chicago: University of Chicago Press, 1994.

CONNOLLY, William. E. Democracy and territoriality. Millennium: journal of international studies, London, v. 20, n. 3, p. 463-84, 1991.

DOBBELAERE, Karel. Secularization: an analysis at three levels. New York: P. I. E. Peter Lang Bruxelles, 2002.

DUYVENDAK, Jan Willem; TONKENS, Evelien; HURENKAMP, Menno. Culturalization of citizenship in the Netherlands. Trabalho apresentado ao 2008 Annual Meeting of the American Political Science Association, Amsterdam, 2008.

DUYVENDAK, Jan Willem; PELS, Trees; RIJKSCHROEFF, Rally (Eds.). A multicultural paradise? The cultural factor in Dutch integration policy. New York: Cornell University Press, 2009.

ENTZINGER, Henk B. Het minderhedenbeleid: dilemma's voor de overheid in Nederland en zes andere immigratielanden in Europa. Meppel: Boom, 1984.

EYERMAN, Ron. The assassination of Theo Van Gogh from social drama to cultural trauma. Durham: Duke University Press, 2008.

GELLNER, Ernest. Nations and nationalism. Ithaca: Cornell University Press, 1983.

GEMEENTE ROTTERDAM. Projectbureau sociale integratie Platform Buitenlanders Rijnmond Sociale integratie . . . zo denken ze erover. Moslims aan het word. Rapport interne debatten, Rotterdam, 2004.

Projectbureau sociale integratie Sociale integratie. . . Rotterdammers in gesprek. Rapport publieke debatten, Rotterdam, 2005.

GHORASHI, Halleh. Ways to survive, battles to win: Iranian women exiles in the Netherlands and the US. New York: Nova Science Publishers, 2003.

GUIRAUDON, Virginie; LAHAV, Gallya. Special issue on immigration policy in Europe: the politics of control. Abingdon: Routledge, 2006. HADDEN, Jeffrey.K. Toward desacralizing secularization theory. Social Forces, Oxford, v. 65, n. 3, p. 587-611, 1987.

HAMMAR, Tomas. Citizenship: membership of a nation and of a state. International Migration, Georgetown, v. 24, n. 4, p. 735-48, 1986.

HERVIEU-LE'GER, Danièle. Space and religion: new approaches to religious spatiality in modernity. International Journal of Urban and Regional Research, Essex, v. 26, n. 1, p. 99-105, 2002.

HOBSBAWM, Eric J. Nations and nationalism since 1780: programme, myth, reality. Cambridge: Cambridge University Press, 1990.

HOUDT, Friso van; SCHINKEL, Willem. Aspecten van burgerschap. Een historische analyse van de transformaties van het burgerschapsconcept in Nederland. B en M: Tijdschrift voor beleid, politiek en maatschappij, Den Haag, v. 36, n. 1, p. 50, 2009.

HUTCHINSON, John. Ethnicity and modern nations. Ethnic and Racial Studies, Oxfordshire, v. 23, p. 651-69, 2000.

IVANESCU, Carolina. Politicised religion and the religionisation of politics. Culture and Religion, Oxon, v. 11, n. 4, p. 309-325, 2010.

JOPPKE, Christian. The retreat of multiculturalism in the liberal state: Theory and policy. The British Journal of Sociology, London, v. 55, n. 2 , p. $237-257,2004$.

KNIPPENBERG, Hans Secularization in the Netherlands in its historical and geographical dimensions. GeoJournal, Den Haag, v. 45, n. 3, p. 209, 1998.

KOOPMANS, Ruud. Contested citizenship: immigration and cultural diversity in Europe. Minneapolis: University of Minnesota Press, 2005. 
LIJPHART, Arend. The politics of accommodation: pluralism and democracy in the Netherlands. Berkeley: University of California Press, 1968.

LLORENT, Vicente; LLORENT, Vicente Jesús. El papel de la mujer en el Cristianismo y el Islam In: ÁLVAREZ, José. Luis; ESSOMBRA, Miquel A. Dioses en las aulas: educación y diálgo interreligioso. Barcelona: Graó, 2009. p. 169-185.

MAHM00D, Saba. Politics of piety: The Islamic revival and the feminist subject. Princeton: Princeton University Press, 2005.

MARTIN, David. A general theory of secularization. New York: Harper \& Row, 1978.

POCOCK, John. The ideal of citizenship since classical times. In: SHAFIR, Gershon. The citizenship debates: a reader. Minneapolis: University of Minneapolis Press, 1998.

RAI, Milan. 7/7: The London bombings, Islam and the Iraq war. London: Pluto, 2006.

Roy, Oliver. Globalised Islam: the search for a new Ummah. London: C. Hurst, 2004.

SCHEFFER, Paul. Het multiculturele drama (the multicultural drama). NRC Handelsblad. Disponivel em: http://retro.nrc.nl/W2/Lab/ Multicultureel/scheffer.html, acesso em: 29, January, 2000.

SCHINKEL, Willem. Denken in een tijd van sociale hypochondrie: Aanzet tot een theorie voorbij de maatschappij. Kampen: Klement, 2008.

SENGERS, Erik. The Dutch and their gods: secularization and transformation of religion in the Netherlands since 1950. Hilversum: Uitg. Verloren, 2005.

SNIDERMAN, Paul. M.; HAGENDOORN, Louk. When ways of life collide: multiculturalism and its discontents in the Netherlands. Princeton: Princeton University Press, 2007.

STARK, Rodney; FINKE, Roger. Acts of faith: explaining the human side of religion, Berkeley and Los Angeles: University of California Press, 2000.

TAMBIAH, Stanley. J. Magic, science, religion, and the scope of rationality. Cambridge: Cambridge University Press, 1990.

TILLY, Charles. Why? Princeton: Princeton University Press, 2006.

VAN DEN BERG, Marguerite; SCHINKEL, Willem. 'Women from the catacombs of the city': gender notions in Dutch culturist discourse. Innovation, Vienna, v.22, n. 4, p. 393-410, 2009.

WILSON, Bryan R.; SOUTH PLACE ETHICAL SOCIETY. The secularization question: is religion losing its social significance? London: South Place Ethical Society, 1996.

WILTERDINK, Nico. Mondialisering, migratie en multiculturaliteit In: GEUIJEN, C. Multiculturalisme. Utrecht: Lemma, p. 55-67, 1998.

YOUNG, Iris M. Justice and the politics of difference. Princeton: Princeton University Press, 1990.

Recebido en: 09.01.2013

Aprovado en: 24.04 .2013

Vicente J. Llorent García es doctor en Pedagogía por la Universidad de Sevilla, licenciado en Ciencias de la Educación con el Primer Premio Nacional concedido por el Ministerio de Educación, profesor de la Universidad de Córdoba (España) y coordinador del Grupo Docente sobre Educación, Diversidad, TIC y Sociedad.

Carolina Ivanescu es investigadora de la Universidad Erasmus en Políticas de Migración e Integración, en la participación de proyectos conjuntos del departamento de Política de la Administración Pública (Grupo de Investigación Pública y Política) y en el departamento de Sociología (Instituto CIMIC). Su experiencia se centra en los movimientos migratorios y estudios sobre la integración e inclusión socioeducativa, análisis comparativos de políticas, técnicas de estudio de casos con un enfoque sobre las minorías y la interacción entre secularidad y ciudadanía. 